Hospital Luis Calvo Mackenna.

Catedra de Fediatria de'. Prof. A. Ariztia,

\title{
PROFILAXIS INTRAHOSPITALARIA CON SULFAMDRAZINA EN LACTANTES
}

Por los Dres. TEOEORO ZENTENO y OSCAR CORREA B.

En los últimos años ha alcanzado amplia difusión en clínica el uso preventivo de sulfonamides en pacientes con enfermedad reumática inactiva ${ }^{l}: i$ is. De la experiencia hasta hoy reunida no parece cater dudas de la alta utilidad de este tipo de profilaxis. el que sin discusión, ha abierto nuevas perspectivas al nifio y adolescente aquejado de dicha afecçión.

El resultado satisfactorio cbtenido con sulfadrogas en el terreno antes mencicnado ha despertads interés por conocer qué grado de protección sería posible cbtener ante otro tipo de infecciones. Coburn', entre otrcs, ha dado a conocer estudios realizados en adultos dirigidos a la prevención de infeccicnes bacterianas de las vías respiratorias superiores. En un alto número de sujetos jóvenes. alistados en la Arma. da de los Estados Unidos, logró obtener. mediante la admı.. nistración profilactica de 1 gramo diario de sulfadazina. una reducción considerable en $\mathrm{l}_{\mathrm{a}}$ incidencia de procesos respitatorics agudos. entre los culales se incluian amigdalitis. faringitis, bronquitis, laringiris, angina de Vincent, neumonía en todas sus formas, escarlatina. faringitis séptica y etrfermedad reumática. Dicho autor señala que en el grupo de control hubc un número doble da consultas médicas por sintomas respiratorios en comparación con el grupo que recítió sulfadiazina, diferencia que fué aún mayor respecto a 
frecuencila de hospitalización, que alcanzó una cifra tres veces superior en los controles. Otro becho que llamó la atención en este trabajo fue el mayor grado de prevención obtenido en infeccicnes de origen estreptocóccico sobre las producidas por otros gérmenes, siendo la incidencia de procesos debido al estreptoccco 11 veces superior en los controles que en los tratados. Watson y colaboradores ${ }^{8}$ han relatado también excelentes resultados en el control de una epidemia de escarlatína con el emplleo profiláctico de sulfadiazina.

En cuanto al riesgo que envuelve el uso prolongado de sulfonamidos, la maycría de los autores están de acuerdo en que él es insignificante. En efecto, tanto las manifestaciones tóxicas como la producción de sensibilización a la droga y desarrollo de resistencia de los gérmenes, se ha visto au: son minimas. Sobre este último punto, por ejemplo, en relación con ef cual muchos han adelantado temores, Norriso ha demostrado, en experiencias realizadas en forma cuidadosa, que, por lo que atañe al estreptccoco bemolítico, este germen no ofrece cambics en sus características bacteriológicas. En la flora de la garganta de individuos sometidos a profilaxis no pudo comprobar aumento ni en la prevalencia de ningún tipo serológico ni en la properción de ellos. Tampocc cbservó modificaciones en la patogenicidad del estreptococo ni en su sensibilidlad a la acción de dosis terapéuticas de sulfadiazina, como to demostraron resultados satisfactorios obtenidos con esta droga en sujetos que contrajeron infecciones estreptocóccicas serias. A este mismo respecto son dignas de hacer notar las conclusiones de Baldwin, quien, en un estudio de control de sujztos reumáticos sometidos anteriormente a prefilaxis de sulfanilamida, demuestra que no se observa aumento de la susceptibilidad a infecciones estreptocóccicas y recidivas rẹumáticas en los años siguientes a la suspensión de la droga.

Es a base de los estudios antes citados que consideramos de-interés investigar la utilidad que pudiera tener el empleo profiláctico de sulfadrogas en lactantes, conocida la relativa alta frecuencia de infecciones que sufre éste y su repercusión sobre el estado nutritivo y salud general. 


\section{Plan de estudio.}

El material de estud!o estuvo constituído por la totalidad de lactantes del Servicio de Asilo del Hospital Luis Calvo Mackenna. Este Servicio, como es de conocimiento. funciona en estrecha conexión con la Casa Nacional dal Niñe. de donde la admisión de los casos está supeditada a causas sociales, lo que hace que la estada de muchos de allos sea a menudo prolengada. El estudio cubrió, como se dijo. todos los lactantes de Servicio, de los que separados por parejas, uno recibió sulfadroga y el otro quedó de control. Salvo pareo lo más estrecho posible reepecto a edad. no se aplicó ningún otro criterio en la realización $d_{e}$ la experiencia, que se inició el 26 de junio de 1947 y se prolongó sin interrupciones hasta el 26 de noviembre del mismo año. Durante el curso de la profilaxis fue nuestra conductia no interferir en forma alguna con el movimiento de altas a in. gresos del Servicio, el cual siguió según rodaje de rigor. Esto fué causa, como puede comprenderse, de que muchos lactantes de ambos grupos no completaran el tiempo total del estudio. Debido a esto y con el fin de poder contar al término del trabajo con grupos, en lo posible, $1_{0}$ más estrechamente comparables, se enrolaron en al todos los lactantes ingresados durante su curso, los que eran asignados aj grupo de tratados o controles, según correspondiera de la ubicación del lactante dado de alta.

En cuanto a la atención dispensada a los niños dutante el tiempo de la experiencia. ella no se modificó de la que como norma se ofrece en el Servicio $y$ todo el Hospital, tanto en lo que se refiere al cuidado individual como a la alimentación, ajustada a los cánones pediátricos corrientes. Con el fin de evitar todo factor de perturbación no se adm:nistraton en el curso del estudio productos patentados de mi nerales $y$ vitaminas $n i$ transfusiones. Respecto a vitamina $D$, no se alteró la conducta puesta en práctica en el niño asilado, que contempla $l_{a}$ administración de 5 gotas dos veces al día de un preparado de concentración corriente, hasta completat 600,000 unidades internacionales, dosis que se repite posteriormente según necesidad. En relación directa con esto mismo debemos hacer presente que no se realizaron movimientos entre el personal de médicos, enfermeras y auxilliares durante el curso del estudio, salvo, naturalmente, los inevitables cambios pasajeros determinados por enfermedades a otras causas. En globo, la experiencia comprendió 
31 lactantes en el grupo que recibió sulfadroga y 26 en el de control; la edad de los cuales iba desde 1 mes a 2 años, a excepción de 2 casos, de 32 y 42 meses. correspondientes a lactantes hipoplásicos, cuyas características físicas $(7,100 \mathrm{y}$ 3.600 gramos, respectivamente), psiquicas $y$ funcionales, los hacen asimilar a la condición de lactantes pequeños.

Como droga se eligió la sulfamerazina (*), que ofrece ventajas sobre la sulfianilamida, sulfatiazol y sulfadiazina, empleadas en otros estudics, debido a su más lenta eliminación. La dosis de medicamento fué de 0.25 gramo para los lactantes con peso superior a 6 kgrs. y de la mitad de ella para los con pesc infericr a dicha cifra. La droga se administró en una sola dosis, dada a las $\mathbf{8}$ horas. Estas dosis. como puede apreciarse, con ligeramente superiores a las empleadas con igral fin en cscclares y adultos, lo que se consideró del caso conocidos los niveles sanguíneos más bajos que se obtienen en la infancia.

A pesar de que las manifestaciones tóxicas, como se dijo, son raras en experiencias de esta indole. se llevó control periódico del recuento de glóbulos rojos y blancos. fórmula leucocitaria, determinación de hemoglobina y exa. men de orina. En la mayoría de los casos se hicieron también determinaciones de la concentración de sulfamerazira en la sangre, realizadas a intervalos variables después de la administración del medicamento.

\section{Resultados y Comentario.}

Estos aparecen expuestos en las tablas y gráfico que se acompañan. En la tabla 1 y gráfico 1 se presentan las diferentes infecciones observadas en ambos grupos durante el tiempo de la experiencia, ordenadas según ș frocuencia. En esta lista se han excluido algunos procesos, entre los que figuran, con su respectiva frecuencia para el grupo de tratados y controles, indicada entre paréntesis, sarampión $(5 \mathrm{y} 6$ ), varicela $(9$ y 8) y vulvovaginitis gonocóccica (3 y 6). Se decidió eliminar las dos primeras enfermedades en atención a su naturaleza etiológica, $y$ la última, por haberse presentado una epidemia de dicha afección en şalas en que la distribución por sexo era desigual, con predominio casi absoluto de lactantes mujeres.

(*) Sulfamerazina Lederke, proporcionada gentilmanta por ia Casa Becker y Saval. 


\section{TABLA N 1}

\section{Incidencia mórbida en 31 lactantes tratados con sulfamerazina y 26 controles.}

Servicio de Aslio. Hospital Lưs Calvo Mackenna. 1947.

\begin{tabular}{|c|c|c|c|c|}
\hline Infecsicnes & Tratados & $\%$ & Controles & $\%$ \\
\hline $\begin{array}{lllllll} & \ldots & \ldots & \ldots & \ldots & \ldots & \ldots\end{array}$ & 38 & 41 & 23 & 18.9 \\
\hline Rinofaringitis .... ... & $\ldots$ & 23.6 & 29 & 23.8 \\
\hline Rinofaringo-bronquitis.$\quad \ldots \ldots \ldots \ldots$ & I] & 11.8 & 18 & 14.8 \\
\hline Otitis congestiva $\ldots \ldots \ldots \ldots \ldots$ & $\ldots$ & $7 . \vec{\jmath}$ & 7 & 5.7 \\
\hline Amigdalitis $\ldots \ldots \ldots \ldots \ldots$ & $\ldots$ & 4.3 & 2 & 1.6 \\
\hline Dispepsia para-enterat $\ldots . . .6 \ldots$ & $\ldots$ & 3.2 & 3 & 2.5 \\
\hline Otitis supurada & $\cdots$ & 2.1 & 2 & 1.6 \\
\hline Abscesos cutáncos y cuero cabelludo ... & $\ldots$ & 2.1 & 8 & 6.5 \\
\hline Bronquitis $\ldots \ldots \ldots$ & $\ldots$ & 1.1 & 4 & 3.3 \\
\hline 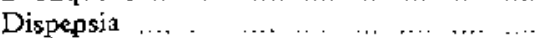 & $\ldots$ & 1.1 & 5 & 4.1 \\
\hline 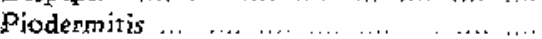 & $\ldots$ & 1.1 & 2 & 1.6 \\
\hline Conjunriviris purulenta $\ldots$... & $\cdots$ & 1.1 & 3 & 2.5 \\
\hline 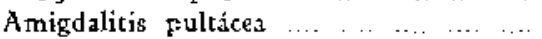 & ... & & 4 & 3.3 \\
\hline 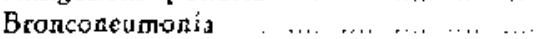 & $\ldots$ & & 3 & 2.5 \\
\hline 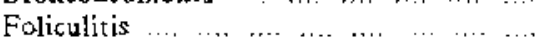 & $\cdots$ & & 3 & 2.5 \\
\hline $\begin{array}{lllllllll}\text { Piuria } & \ldots & \ldots & \ldots & \ldots & \ldots & \ldots \\
\end{array}$ & $\ldots$ & & 2 & 1.6 \\
\hline $\begin{array}{llllllll}\text { Enterocolitis } & \ldots & \ldots & \ldots & \ldots & \ldots & \ldots & \ldots\end{array}$ & $\ldots$ & & 2 & 1.6 \\
\hline Adenitis cervical supurada $\ldots . . . . .$. & $\ldots$ & & 2 & 1.6 \\
\hline $\begin{array}{llllllll}\text { Totales } & \ldots & \ldots & \ldots & \ldots & \ldots & \ldots & \ldots\end{array}$ & 93 & 100 & 122 & 100 \\
\hline
\end{tabular}

En la tabla 2 se da a conocer el resultado del estudio estadístico del material. Se adoptó, según puede observarse. el término de personas-semana, con el fin de indicar con él de una manera exacta el tiempo de exposición al riesgo de enfermar que corrieron independientemente cada uno $\mathrm{d}_{2}$ los individuos de los distintes grupos sujetos a la experiencia. Como puede aprecilatse en las cifras of recidas en esta tabla. este riesgo fué maycr. en 40.69 personas-semana (518.32. $477.83)$, en el grupe de los individuos tratados con sulfamerazina. La menor duración de la experiencia o el menor número de personas'semana que integraron al grupo de los cantroles no deja de sar indiferente para el grupo de los tratados, puesto que es evidente que un mayor número de fersonas expuestas y sujetas a las condicicnes señaladas en nuestro estudic, deben necesariamente correr un riesgo de enfermedad mayor.

Las Tasas Crudas de mcrbilidad, es decir, la relación entre el número de cuadros nosológicos durante un período determinado de tiempo sobre el total de niños expuestos al 
riesgo de enfermar durante el mismo perícdo multiplicado por 1,000, acusa una incidencia menor de casos en el grupo de tratados con sulfamerazina $(179.35 \%)$ que en el de controles $(255.32 \%)$. Este resultado, sin embargo, no podía, en nuestro caso, ser considerado como definitivo, ya que debíamos eliminar la influencia posible que sobre él pudiera derivar de la distinta composición de grupos del mate-

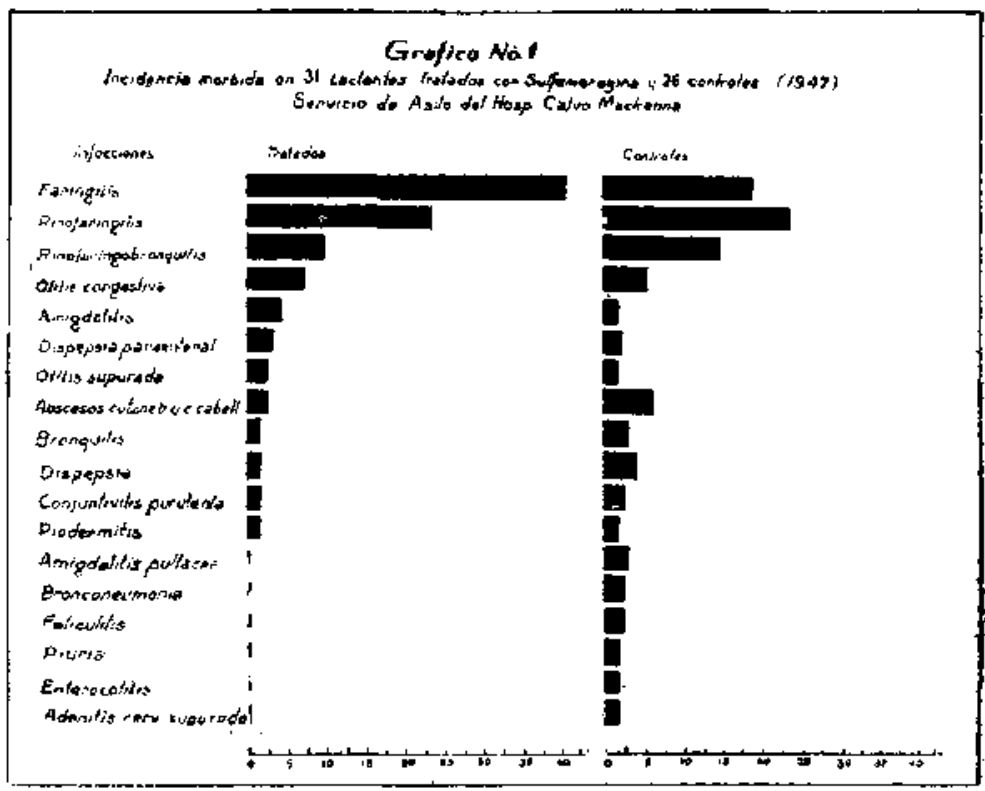

rial en lo que a edad y número de personas expuestas se refiere, para cuyo objeto se procedió al cálculo de las Tasas Ajustadas. En la tabla mencionada puede verse, en efectó, que mientras el $22.5 \%$ de los niños idel grupo de los tratados pertenece al primer trimestre de la vida, sólo el $7.64 \%$ de los niños controles se encuentran en este grupo de edad.

Las Tasas Específicas de morbilidad por edad, o sea, la relación entre la incidencia de los casos registrados en cada grupo de edad sobre el número de personas-semana de individuos expuestos al riesgo de enfermar multiplicada po: 1,000 , demuestra que el riesgo es evidentemente menor in los tratados $(201.3 \%)$ que en los controles $(326.7 \%)$. Este resultado tiene aún mayor valor si se considera que es más alto el número de personas-semana expuestas al riesgo de enfermar en el primer trimestre entre los primeros (109.28) que entre los segundos (42.85), en el supuesto que 
el medicamento administrado fuese del todo indiferente y ia incidencia amagara por igual a las persenas de ambos grupos.

$\mathrm{Si}$ aplicamos las $T$ asas Específicas de morbilidad por edad de cada uno de los distintos grupos analizados, tratados y contrcles, a una población tips, con el objeto de determinar cuál hubiera sido el número de personas afectadas en esta nueva población si en ella se bubiera dejado de sentín la fuerza de morbilidad que afectó independientemente a los tratados y controles en sus diferentes grupos etarios, obtendríamcs, en términos teóricos, el número de individuos que enfermarian en esta nueva población. Ahora, la suma de estas cifras, que reptesentan individuos, puestas en relación con la población standard, nos permitiría conocer la relación existente entre ambas fuerzas de morbilidad, que, aun que actuando en forma independiente, manifestarian su vetdadera potencialidad reflejada de una manera precisa frente a los individuos de una población comin. En nuestros cálculos hemos adoptado como población standard la suma de las ciftas que integran las personas-semana de cada uno de los distintos grupos de edades. Con las cifras así obtenidas, transformadas en tasas (Tasas Ajustadas), hemos podido precisar que existe una tasa de mayor morbilidad en los individuos que pertenecen al grupo de los controles $(274.63 \%)$, con evidente ventaja para los tratados $(181.34 \%)$, becho que no era posible deducir de la ins pección simple o del estudic superficial que se deriva de ila expresión numérica de las Tasas Crudas $(255.32 \%$ y $179.35 \%$, respectivamente).

Las Tasas Crudas de morbilidad (relación entre el número de enfermadades presentadas sobre el total de personassemana multiplicada por 100) dan para al grupo de lọ tratados una incidencia de $17.93 \%$ y para los controles de $25.53 \%$ y las tasas ajustadas valores $d_{e} 18.13 \%$ y $27.46 \%$. respectivamente. La diferencia entre estos últimos porcentajes es de $9.33 \%$, cifra que es superior a la observada en el cálcu lo hecho a base de las tasas crudas $(7.6 \%$ ) y sobre la cual es necesario penetrar en su significado estadistico. La D. S. de $18.13 \%$ del grupo de los tratados es igual a $\sqrt{ } 2.8625$. la de $27.46 \%$ del grupo dé los controles a $\sqrt{ } 4.1687$ y la D. S. de la diferencia de ambos porcentajes a $\sqrt{2.8625}+$ 4.1687 , esto es, a $V 7.0312$, operación cuyo resultado (sigma) es igual a 2.65. Ahora, la diferencia entre ambos porcentajes, $9.33 \%(\mathrm{X})(27.46-18.13)$, medida en términos de la D. S. de la diferencia entre ellos, 2.65 (b), es igual 
a $3.5(\mathrm{X} / 6)$, valor que como expresión en el análisis estadís. tico es de alta y significativa importancia ${ }^{\prime \prime}$.

En efecto, el cálculo de las probabilidades (T:blas de 11 probabilidad integral) ${ }^{11}$ permite deducir, que un hecho de la naturaleza dei antes mencionado, una diferencia de $9.33 \%$, podriamos observarlo como fruto de la casualidad 4 vacos en 10,000 ensayos semejantes. En otros términcs. si nos fuera

$$
\text { TABLA N" }
$$

\begin{tabular}{|c|c|c|c|c|c|c|c|c|c|c|c|}
\hline \multicolumn{12}{|c|}{ 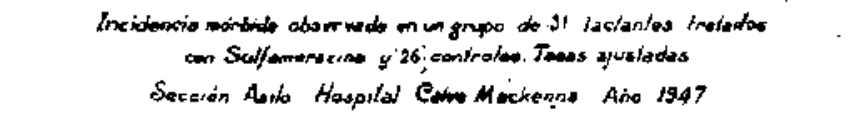 } \\
\hline \multirow{2}{*}{ thed } & \multicolumn{4}{|c|}{ 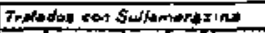 } & \multicolumn{3}{|c|}{ Coneroies } & & \multirow{2}{*}{ standionos } & \multicolumn{2}{|c|}{ Nemproded deramen } \\
\hline & N & and & 5 & Ex & meth & A & $i_{t}+\theta_{i}$ & & & Pendoder & Centroler \\
\hline $\begin{array}{l}15 \\
3 \cdot 3 \\
0.6 \\
3-11 \\
12 \cdot 20 \\
23\end{array}$ & $\begin{array}{l}7 \\
3 \\
8 \\
4 \\
0\end{array}$ & $\begin{array}{r}100.28 \\
11.42 \\
162.05 \\
65.48 \\
128.58\end{array}$ & $\begin{array}{l}22 \\
10 \\
24 \\
11 \\
26\end{array}$ & $\begin{array}{l}201.31 \\
194.47 \\
44.37 \\
105.61 \\
202.45\end{array}$ & $\begin{array}{l}2 \\
4 \\
8 \\
3 \\
7 \\
2\end{array}$ & $\begin{array}{c}42.85 \\
15.71 \\
145.71 \\
40.71 \\
120 . \\
42.80\end{array}$ & $\begin{array}{l}14 \\
26 \\
33 \\
17 \\
27 \\
5\end{array}$ & $\begin{array}{l}326.72 \\
303.4 \\
226.47 \\
417.58 \\
225 . \\
116.68\end{array}$ & $\begin{array}{l}152 \\
137 \\
309 \\
107 \\
249\end{array}$ & $\begin{array}{l}31 \\
27 \\
40 \\
18 \\
91\end{array}$ & $\begin{array}{l}50 \\
42 \\
70 \\
44 \\
56\end{array}$ \\
\hline الهاdT & $\sqrt{31}$ & 51852 & 95 & & $2 \theta$ & 47.63 & 122 & & 934 & 175 & 262 \\
\hline 70 & & 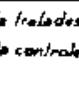 & & $\begin{array}{l}179.35 \% \\
255.32 \%\end{array}$ & & $\begin{array}{l}\text { TAd of } \\
\text { Tats a }\end{array}$ & oste & $6 \mathrm{~cm}$ & & $\begin{array}{l}1.34 \% \\
4.63 \%\end{array}$ & \\
\hline
\end{tabular}

dado repetir esta experiencia en las mismas condiciones planteadas en nuestro trabajo, sujetas al mismo período de obser vación y la droga no dejase de ser indiferente, obtendríamos una diferencia en los porcentajes igual o superior a la encontrada entre ambos grupos en apenas 4 veces en 10,000 ensayos sucesivos, como resultado de las variaciones del azar. Si se pretende aceptar este hecho como posible, se debe reconocer, sin embargo, que su producción se halla sujeta a una probabilidad muy remota y se debe concluir, entonces, que lis droga modifica de una manera substancial la situación de uno de los grupos sujetos a la experiencita en el sentido que evita, previene o disminuye en parte el riesgo del individuo expuesto a una determinada enfermedad.

Respecto a reacciones tóxicas de la sulfamerazina, debemos hacer presente que ellas no se observaron en el curso del estudio. En efecto, no se preszntaron mànifestaciones cutáneas. fiebre de droga, vómitos ni otros signos y sintomas que se describen durante la administración de sulfonamidos. En cuan. 
to a alteraciones del hemograma, en ningún caso tvimos esta. telecerse anemia ni tampoco descensos del recuento de glóbu. los blances a límites patológicos. En un lactante en quien antas de la iniciación de la experiencia existia un recuento de 6,400 , éste descendió durante los dos primeros mespes de profilaxis a 4.600, para postericrmente ascender en forma esFontánea a 7,200, cifra alrededor de la cual sz mantuvo en adelante. En este paciente, por lo demás, el porcentaje de granulccites en ningún mcmento hizo pensar en efecto tóxi co de la droga, ya que de $57 \%$ que existían al comienzo, descendieron a $49 \%$, para postericrmente subir a $72 \%$. En los cascs restantes tampcco se observó nada anormal en el número de ellos y así, al lado de lactantes que mantuvieron o aumentaron su cifra. hubo otros en los que se acusó ligero descensc. A cste respecto $\varepsilon \varepsilon$ digno de mención un caso en que de 8 granulocitos que presentaba al empezar el estudio (recuento de 8.800), ellos ascendieron a 17 a los dos meses y a 20 al finalizar la experiencia, sin evidenciarse casi cambios en el número total de elementos blancos $(8,200)$.

Igual cbservación cabe referente al efecto de la sulfamerazina sobre las vías urinarias. Salvo indicios de altúmina y escasos glóbulos rojos encontrados en forma pasajera en algunos casos, en la mayoria el examen de orina fué a repetición totalmente negativo.

La determinación de la sulfamerazina se hizo, como se declaró, a intervales variables después de la administración de la droga. En muestras obtenidas minutos antes de dar la dosis diaria, es decir, casi 24 horlas después de la ingestión del medicamento, se comprobaron niveles sanguíneos que iban de indicios en algunos, la minoria, a concentraciones de $0.5,1$, 1.5 y 2 miligramos por ciento, en el resto. Niveles más altos, variables entre 2 a 5 miligramos, se encontraron en determinaciones realizadas 4 horas después de la administración de It droga y cifras ligeramente más bajas, entre 2 a 4 miligna. mos, a las 8 horas de aquélla. Se hicieron extracciones a estos dos íltimos intervalos, con el fin de conocer las concentraciones más altas a que pudiera llegar la droga. conocido el estudio de Forbes y colaboradores ${ }^{12}$, en que se demuestra que bajo los dos años, el nivel sanguíneo máximo de la sulfamerazína, cuando se administra per os, se alcanza a alrededor de las 4 horas y sobre dicha edad entre las 6 y 8 horas.

Finalmente, otro punto al cual debemos también referirnos, es al comportamiento de los casos bajo profilaxis frente 
a dosis terapéuticas de sulfonamidos. Cuando recién se inició la administración profiláctica de sulfadrogas en la enfermedad reumática inactiva se abrigaron serjos temores de que con el uso continuado del medicamento sa crearan condiciones favorables para el desarrollo de resistencia de algunas especies microbianas en forma tal que el empleo de dosis terapéuticas corrientes de él no se acompañara de respuesta clínica. Ya en un comienzo hicimos ver, a base de la opinión de varios autores, la rareza de estra posibilidad, lo que se confirmó en nuestro ectudio. En efecto, en ninguno de los numerosos casos que debieron ser sometidos a trataminnto con sulfonamidos según pauta acostumbrada, debid ${ }_{C}$ a la aparición de infecciones de importancia, se observó respuesta que pudiera hacer sospechar el desarrollo de resistencia mictobiana.

\section{Resumren y Conclusión.}

Se da cuenta de un estudio sobre uso profiláctico de la sulfamerazina, en un grupo de 31 lactantes (518.52 personas-semana), cuya evolución se compara con la de 26 (477.83 personas-semana) que sirvieron de control. La experiencia abarcó un período de 5 meses, tiempo durante el cual tanto los niños tratados como los controles estuvieron sujetos a iguales condiciones de ambiente, alimentación y atención médica y de enfermeria. La droga se administró en dosis de 0.25 gramo al día, dada de uria vez, en lactantes con peso superior a 6 kgrs. y de la mitad de ella en aquéllos con peso inferior a dicha cifra y tanto en unos como en otros se llevó un contrel cuidadoso clínico y de laboratorio, incluido en esto último hemograma y examen de orina. hechos:

Del análisis del material se desprenden los siguientes

1. La droga fué bien tolerada y no se observaron signos ni síntomas de toxicidad durante su administración.

2. Durante el tiempo de la experiencia, se comprobó una incidencia menor de infecciones en los tratados $(18.13 \%$ ) que en los controles $(27.46 \%)$, diferencia que es estadísticamente significativa $(\mathrm{X} / 6=3.5)$.

3. La suliamerazina, empleada en las condiciones expuestas en el trabajo, demuestra ser eficaz en disminuir las tasas de incidencia de un determinado tipo de infecciones en un grupo de niños hasta los dos años de edad. 


\section{Summary and Conelusion.}

An experimental study on the prophylactic use of sulfamerazine in infants is reported. The work embraced 31 infants (518.52 patient weeks) to whom sulfamerazine was given ( 0.125 and $0.25 \mathrm{gm}$., according to the body weight) in a single daily dose and 26 infants $(477.83$ patient weeks). taken as controls. The study was extended throughout fico months, during which time the children of both groups were under the same environmental, feeding and medical and nursing care conditions. lowing:

The analysis of the work permits to point out the fol-

I. Sulfamerazine was well tolerated and no toxic reactions were encountered.

2. It was observed a lower incidence of infections in the treated group $(18.13 \%)$ than on the control one $(27.46 \%)$, difference which is statistically significant $(\mathrm{X} / \mathrm{t}=3.5)$.

3. Sulfamerazine, given under the conditions settled in the study, demonstrates to reduce the rates of incidence of various kinds of infections in a group of children up to two years of age.

\section{Bibliografía.}

1. COOBURN. A. Y MOORE, L. -. The prophylactic use of sulfanilamida in streptococcal respiratory infections, with special reference to rhecmatic fever. J. Clin. Investigation 18: 147, enero de 1939.

2.-THOMAS, C.: FRANCE. R. y REICHSMAN. F. - The prophylitic use of sulfanilamide in patients susceptible to rbeumatic fever. J. A. M. A. 116:551.560.15 de febrero de 1941.

3.-DODGE, K. BALDWIN, J, y WEBER, M. — The prophylactic use of sulfanilamides in children with inactive rheumatic fever. J, Pediat. 24: 483.501, mapo de 1944.

4.-WOLF, R.; RAUH, L. y LYON, R. - The prevention of rhenmati: recurrences in children by the use of sulfathiazole and sulfadiazine. J. Pcdiat. 27: $\overrightarrow{2} 16-520$, diciembre de 1945.

5.-BALDWIN. J. - Follow-up study in rheumatic subjects previous'.y treated with prophylactic sulfanilamide. J. Pediat. 30:67.71. enero de 1947.

6.-BALDWIN, J. -- Sulfadiazine prophylaxis in children and adolessents with inactive rheumatic fever. J, Pediat. 30: 284-288, marzo de 1947. 